\title{
Alterações gengivais em crianças a efetuar medicação anti-epiléptica 4 anos de avaliação clínica
}

\author{
Ivo A. Furtado* \\ Paulo A. Palmela** \\ Virgínia B. Milagre**
}

\section{RESUMO}

Os autores efetuaram durante 4 anos a avaliaçāo clínica das Alteraçōes Gengivais em Crianças a fazer medicação anti-epiléptica. Foram seguidas 300 crianças de risco médico acrescido, de ambos os sexos, até aos 12 anos de idade; tomaram como grupo testemunha igual número de crianças saudáveis, da mesma faixa etária.

Analisaram a distribuição das crianças consoante o sexo e a idade, em ambos os grupos. Verificaram o número de casos de crianças com epilepsia e a data do diagnóstico, início da terapêutica e ainda o fármaco utilizado.

Determinaram a prevalência de hiperplasia gengival nos diferentes grupos e a sua correlaçảo com a medicaçāo, a higiene oral e o tipo de dieta. Fizeram propostas terapêuticas com base na experiência que obtiveram.

Concluíram que a hiperplasia gengival se encontra predominantemente associada à Fenitoína e à Primidona; apresenta também uma íntima relaçāo com a deficiente higiene oral e com a dieta mole.

\section{SUMMARY}

The authors have made during four years, the clinical evaluation of the gingival alterations in the children getting anti-epileptic medication.

A follow upof three hundred increased medical risk children, was done. These children, from both the sexes were aged until twelve.

An equal number of healthy children, aging the same range, was considered as testimony group.

The distribution of these children was analysed according the sex and the age in both the groups.

The number of the cases of children with apilepsy and the time of the diagnosis, the therapeutic beginning and still the used medicine, was verified.

The prevalence of gingival hyperplasia at the different groups and its correlations with the medication, the oral hygiene and the type of the diet were recorded.

Therapeutical proposals based on the obtained experience were advanced.

The authors have concluded that the gingival hyperplasia is mainly associated to Phenytoin and Primidone and has also a close connection with the poor oral hygiene and the soft diet.

\section{DESCRITORES}

\author{
EPILEPSIA • ANTI-EPILÉPTICOS • HIPERPLASIA GENGIVAL • DIETA MOLE • HIGIENE ORAL
}

\section{INTRODUÇÃO}

As epilepsias são um grupo de distúrbios caracterizados por alteraçōes paroxísticas crônicas e recorrentes da função neurológica, causadas por anomalias da atividade elétrica do cérebro. Săo distúrbios neurológicos comuns e considera-se que afetam 0,5 a $2 \%$ da população, podendo ocorrer em qualquer idade (1) (2).

$\mathrm{Na}$ terapêutica destas situaçōes, recor- re-se a um grupo de fármacos depressores seletivos do S.N.C., que permitem evitar e controlar a frequência das crises convulsivas - medicamentos anti-epilépti$\cos (3)$.

Estes classificam-se em Barbitúricos (Fenobarbital e Primidona), Hidantoína (Fenitoína), Oxazolidonas, succinimidas (etosuccimida) e outros (Carbamazepina, Valproato de sódio, clonazepam, etc.) (3) (1).
Atualmente os mais usados na clínica

* Médico Estomatologista Responsável pela Consulta de Estomatologia Pediátrica do Hospital de Santa Maria - Lisboa - Portugal. Professor de Medicina Dentária Preventiva e Odontopediatria do Instituto Superior de Ciências da Saúde - Lisboa - Portugal.

** Médico Interno do Internato Complementar de Estomatologia do Hospital de Santa Maria - Lisboa.
R. Fac. Odontol.

Porto Alegre
V. 32

N. 1

p.27-31

JULHO

1991 
săo o Fenobarbital, a Primidona, Fenitoína, Carbamazepina, Valproato de Sódio e Clonazepam.

Estes fármacos apresentam açōes acessórias, que devem ser conhecidas dos profissionais que lidam com os pacientes epilépticos. Assim, são de salientar as mais frequentes, como a vertigem, a sonolência, a ataxia, as náuseas, os tremores, as discrasias hemorrágicas, diptopia, as disritmias cardíacas, bem como a hiperplasia gengival.

A Hiperplasia Gengival, tem sido principalmente associada ao uso de fenotoína e de primidona (4) (5). (Fig. 1).

A Hiperplasia Gengival consiste numa proliferaçăo do tecido conjuntivo fibroso com numerosos fibroblastos, sem componente inflamatório evidente (6).

Como causa tem sido apresentada a ativação dos mastócitos locais com libertaçāo de mediadores químicos que ativam os fibroblastos (4).

Muitos autores (4) concordam que fatores inflamatórios locais desempenham também importante papel na etiopatogenia da hiperplasia gengival.

Reconhece-se ainda uma correlaçăo entre $\circ$ grau de hiperplasia e a dose diária de anticonvulsivante, a má higiene oral, outros irritantes locais e a idade do paciente.

A presença de dentes é indispensável ao desenvolvimento da hiperplasia; as zonas desdentadas são, por norma, poupadas $(5,7)$.

\section{MATERIAL E MÉTODOS}

O Universo do nosso estudo abrangeu 300 crianças de risco médico acrescido, de ambos os sexos e idades até aos 12 anos inclusive, utentes da Consulta de Estomatologia Pediátrica do Hospital de Santa Maria (H.S.M.).

O grupo testemunha incluiu igual número de crianças do mesmo grupo etário e utentes da Consulta Geral de Estomatologia do H.S.M.

Estas crianças foram acompanhadas de $1^{\circ}$ de Julho de 1986 a 30 de Junho de 1990.

$\mathrm{Na}$ distribuiçăo das crianças dos dois grupos por idade e sexo, encontramos praticamente uma sobreposição dos valores percentuais (Quadros $1 \in 2$ ), bem como uma predominância dos grupos etários mais velhos (Gráficos 1 e 2).

Consideramos como sub-universo, o grupo das crianças epilépticas, tendo encontrado 51 casos, que representam $17 \%$ do Universo. $\mathrm{Na}$ análise da sua distribuição por idade e sexo constatamos maior frequência dos grupos etários mais velhos (Quadro 3 e Gráfico 3).

Apontamos 3 razöes para este fato:

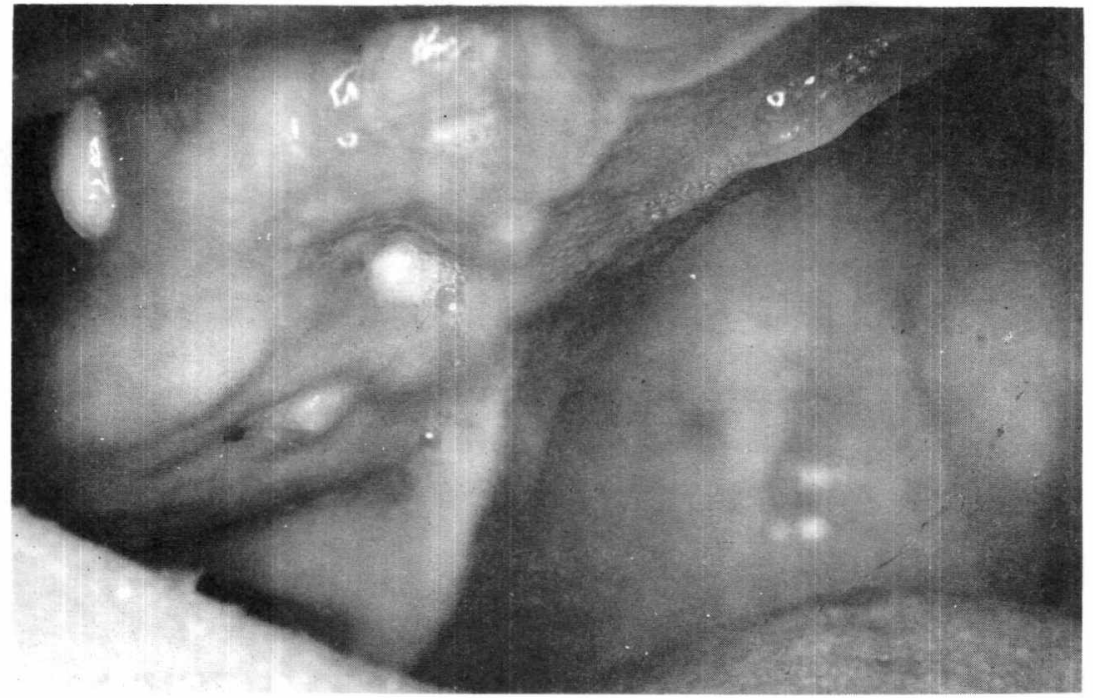

QUADRO 1: Distribuiçãa das crianças por idade e sexo Consulta đe Estomatologia Pediátrica

\begin{tabular}{|c|c|c|c|c|}
\hline \multirow{2}{*}{\begin{tabular}{c}
\multirow{2}{*}{ IDADE } \\
(AnOs)
\end{tabular}} & \multicolumn{3}{|c|}{$\begin{array}{c}\text { NÚMERO DE } \\
\text { CRIANÇAS OBSERVADAS }\end{array}$} & $\begin{array}{c}\text { PERCENTAGEM DE } \\
\text { CRIANÇAS OBSERVADAS }\end{array}$ \\
\cline { 2 - 5 } & SEXO $\sigma^{*}$ & SEXO $\sigma^{*}$ & SEXOS $\sigma^{*}+\sigma^{*}$ & SEXOS $\sigma^{2}+\sigma^{*}$ \\
\hline$<1$ & 0 & 1 & 1 & 0,33 \\
1 & 0 & 0 & 0 & 0,00 \\
2 & 1 & 1 & 2 & 0,67 \\
3 & 2 & 7 & 9 & 3,00 \\
4 & 6 & 6 & 12 & 4,00 \\
5 & 16 & 6 & 22 & 7,33 \\
6 & 18 & 15 & 33 & 11,00 \\
7 & 20 & 11 & 31 & 10,33 \\
8 & 24 & 26 & 50 & 16,67 \\
9 & 15 & 21 & 36 & 12,00 \\
10 & 22 & 17 & 39 & 13,00 \\
11 & 12 & 11 & 23 & 7,67 \\
12 & 20 & 22 & 42 & 14,00 \\
\hline TOTAIS & 156 & 144 & 300 & 100 \\
\hline
\end{tabular}

QUADRO 2: Distribuição das crianças por idade e sexo Consulta Geral de Estomatologia Grupo Testemunha (Controle)

\begin{tabular}{|c|c|c|c|c|}
\hline \multirow{2}{*}{$\begin{array}{l}\text { IDADE } \\
\text { (Anos) }\end{array}$} & \multicolumn{3}{|c|}{$\begin{array}{c}\text { NÚMERO DE } \\
\text { CRIANÇAS OBSERVADAS }\end{array}$} & $\begin{array}{l}\text { PERCENTAGEM DE } \\
\text { CRIANÇAS OBSERVADAS }\end{array}$ \\
\hline & SEXO O" & SEXO O & SEXOS $\sigma^{a}+\sigma^{\circ}$ & SEXOS $\sigma^{\prime \prime}+\sigma^{*}$ \\
\hline$<1$ & 2 & 2 & 4 & 1,33 \\
\hline 1 & 4 & 3 & 7 & 2,33 \\
\hline 2 & 4 & 5 & 9 & 3,00 \\
\hline 3 & 4 & 4 & 8 & 2,67 \\
\hline 4 & 6 & 7 & 13 & 4,33 \\
\hline 5 & 13 & 15 & 28 & 9,33 \\
\hline 6 & 9 & 5 & 14 & 4,67 \\
\hline 7 & 5 & 15 & 20 & 6,67 \\
\hline 8 & 20 & 23 & 43 & 14,33 \\
\hline 9 & 23 & 23 & 46 & 15,33 \\
\hline 10 & 11 & 18 & 29 & 9,67 \\
\hline 11 & 11 & 19 & 30 & 10,00 \\
\hline 12 & 31 & 18 & 49 & 16,40 \\
\hline TOTAIS & 143 & 157 & 300 & 100 \\
\hline
\end{tabular}




\section{DISTRIBUIÇÃO DAS CRIANÇAS SEGUNDO A IDADE CONSULTA DE ESTOMATOLOGIA PEDIÁTRICA}

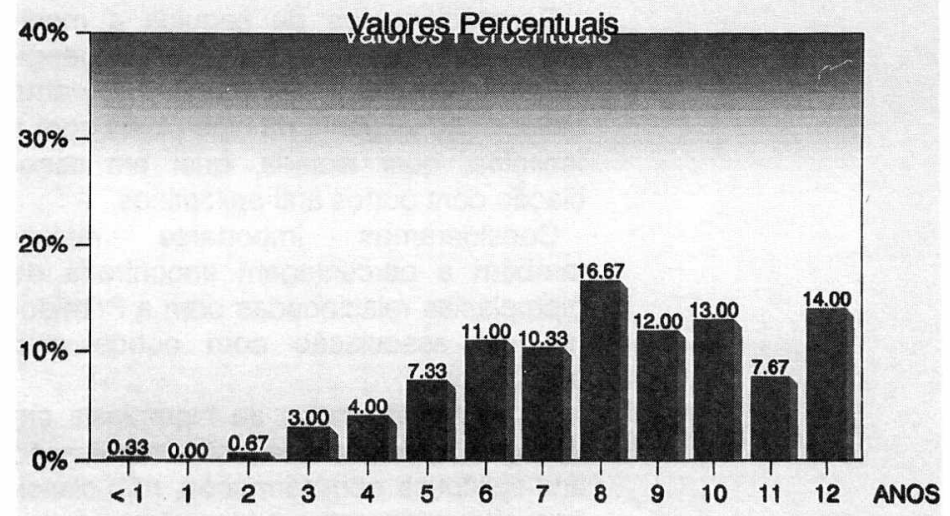

DISTRIBUIÇÃO PERCENTUAL DAS CRIANÇAS EPILÉPTICAS SEGUNDO A IDADE

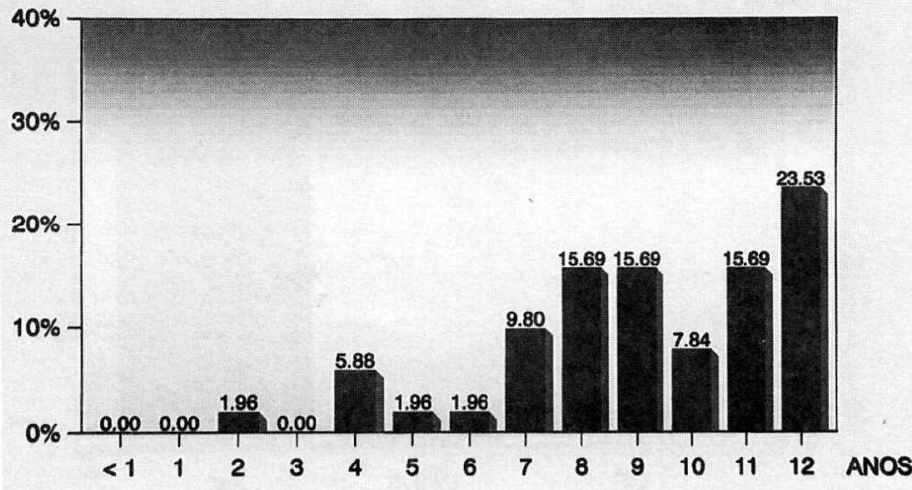

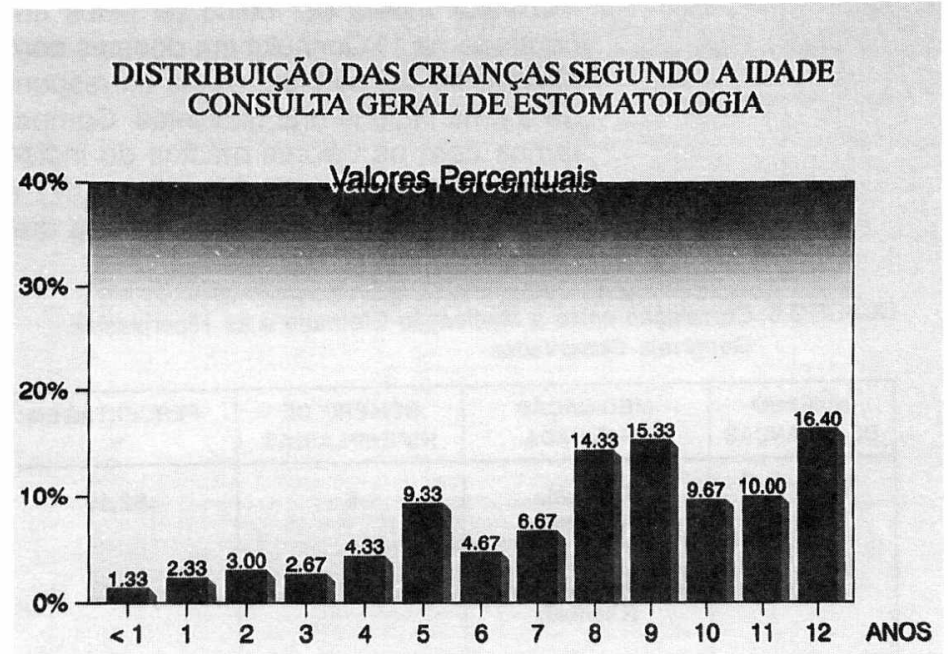

QUADRO 3: Distribuição das crianças Epilépticas Por Idade e Sexo

\begin{tabular}{|c|c|c|c|c|}
\hline \multirow{2}{*}{$\begin{array}{c}\text { IDADE } \\
\text { (AnOs) }\end{array}$} & \multicolumn{2}{|c|}{$\begin{array}{c}\text { NÚMERO DE } \\
\text { CRIANÇAS OBSERVADAS }\end{array}$} & $\begin{array}{c}\text { PERCENTAGEM DE } \\
\text { CRIANÇAS OBSERVADAS }\end{array}$ \\
\cline { 2 - 5 } & SEXO $\sigma^{7}$ & SEXO $\sigma^{*}$ & SEXOS $\sigma^{7}+\sigma^{*}$ & SEXOS $\sigma^{7}+\sigma^{*}$ \\
\hline$<1$ & 0 & 0 & 0 & 0,00 \\
1 & 0 & 0 & 0 & 0,00 \\
2 & 0 & 1 & 1 & 1,96 \\
3 & 0 & 0 & 0 & 0,00 \\
4 & 3 & 0 & 3 & 5,88 \\
5 & 0 & 1 & 1 & 1,96 \\
6 & 1 & 0 & 1 & 1,96 \\
7 & 2 & 3 & 5 & 9,80 \\
8 & 3 & 5 & 8 & 15,69 \\
9 & 3 & 5 & 8 & 15,69 \\
10 & 1 & 3 & 4 & 7,84 \\
11 & 4 & 4 & 8 & 15,69 \\
12 & 4 & 8 & 12 & 23,53 \\
\hline TOTAIS & 21 & 30 & 51 & 100 \\
\hline
\end{tabular}

1. A distribuição etária do próprio Universo

2. O envio destes doentes à nossa Consulta, após controle da sua patologia médica de base

3. Serem inicialmente seguidos em ambulatório e enviadas posteriormente à nossa consulta pelas características específicas da sua patologia.

Para a realização do nosso trabalho recolhemos elementos da história clínica de todos os pacientes, dando relevo ao diagnóstico de epilepsia referenciando a data de início das convulsōes, a medicação efetuada e respectiva dosagem; determinamos o índice de placa (Ramfjord) e procedemos ao registro e a análise da dieta.

\section{RESULTADOS}

Diagnosticamos 16 casos de hiperplasia gengival, sendo 1 caso no grupo testemunha, 2 casos em crianças doentes não epilépticas e 13 casos em crianças epilépticas. (Quadro 4 e Gráfico 4).

QUADRO 4: Número e Percentagem de Hiperplasias

\begin{tabular}{|c|c|c|}
\hline & NỦMERO & PERCENT AGEM \% \\
\hline $\begin{array}{c}\text { Crianças } \\
\text { Saudáveis } \\
n=300\end{array}$ & 1 & 0,33 \\
$\begin{array}{c}\text { Crianças Doentes } \\
\text { não Epilépticas } \\
n=249\end{array}$ & 2 & 0,80 \\
$\begin{array}{c}\text { Crianças } \\
\text { Epilépticas } \\
n=51\end{array}$ & 13 & 25,49 \\
\hline
\end{tabular}




\section{GRÁFICO COMPARATIVO DA PERCENTAGEM DE \\ HIPERPLASIAS GENGIVAIS EM CRIANÇAS EM \\ $100 \%$ \\ DIFERENTES SITUAÇÕES}

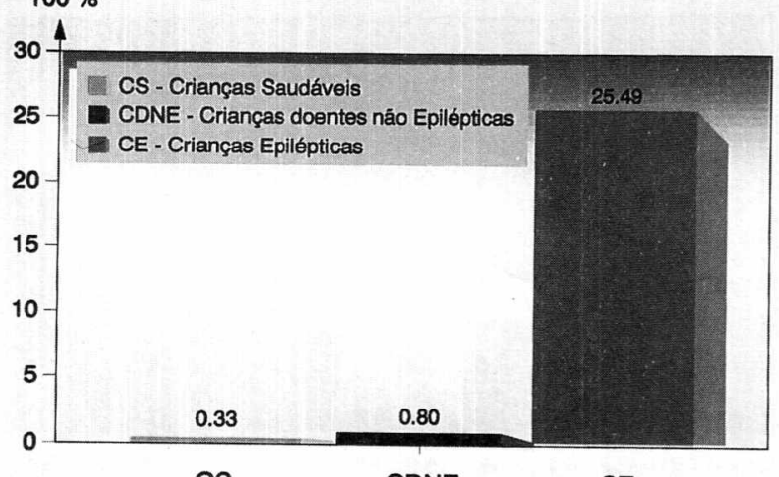

CS

CDNE

CE

QUADRO 5: Prevalência de Hiperplasia Gengival em Crianças Medicadas com Anti-Epilépticos

\begin{tabular}{|c|c|c|c|c|}
\hline \multirow{2}{*}{$\begin{array}{c}\text { IDADE } \\
\text { (AnOs) }\end{array}$} & \multicolumn{3}{|c|}{$\begin{array}{c}\text { NÚMERO DE CRIANCAS } \\
\text { COM HIPERPLASIA }\end{array}$} & $\begin{array}{c}\text { PERCENT. DE CRIANÇAS } \\
\text { COM HIPERPLASIA }\end{array}$ \\
\cline { 2 - 5 } & SEXO O & SEXO O & SEXOS O + O & SEXOS O + O \\
\hline 1 & 0 & 0 & 0 & 0,00 \\
1 & 0 & 0 & 0 & 0,00 \\
2 & 0 & 1 & 1 & 7,69 \\
3 & 0 & 0 & 0 & 0,00 \\
4 & 1 & 0 & 1 & 7,69 \\
5 & 1 & 0 & 1 & 7,69 \\
6 & 0 & 1 & 1 & 7,69 \\
7 & 0 & 1 & 1 & 7,69 \\
8 & 1 & 1 & 2 & 15,39 \\
9 & 0 & 1 & 1 & 7,69 \\
10 & 0 & 0 & 0 & 0,00 \\
11 & 1 & 0 & 1 & 7,69 \\
12 & 1 & 3 & 4 & 30,78 \\
\hline TOTAIS & 5 & 8 & 13 & 100 \\
\hline
\end{tabular}

\section{PREVALÊNCIA DE HIPERPLASIA GENGIYAL}

\section{EM CRIANÇAS MEDICADAS COM ANTI-EPILEPTICOS}

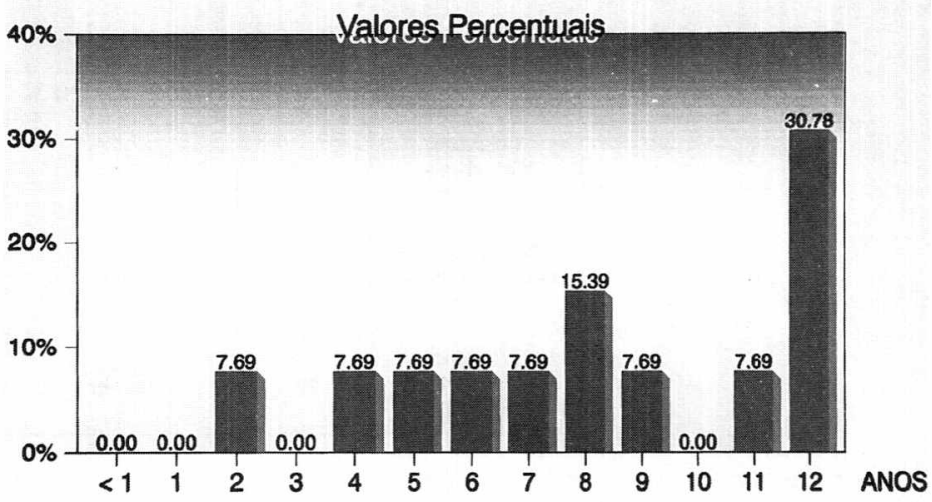

O Quadro e o Gráfico número 5, mostram a prevalência da hiperplasia gengival em crianças medicadas com anti-epilépticos.

Correlacionamos de seguida a medicação efetuada e as hiperplasias gengivais observadas, tendo encontrado uma associação evidente da hiperplasia com a fenitoína, quer isolada, quer em associaçăo com outros anti-epilépticos.

Consideramos importante realçar também a percentagem encontrada de hiperplasias relacionadas com a Primidona, em associaçảo com outros antiepilépticos.

Os restantes casos de hiperplasia em crianças a efetuar atualmente medicaçāo anti-epiléptica com fármacos, nāo classicamente associados à hiperplasia, todos eles tinham feito anteriormente medicaçāo com Fenitoína. (Quadro 6).

0 valor médio do índice de placa encontrado na $1^{a}$ Consulta em doentes com hiperplasia, foi de 2,52 , o que corresponde a uma higiene oral deficiente. Comparamos com os valores médios do índice de Placa do grupo testemunha $(1,67)$ e do grupo das crianças com doença que näo a epilepsia $(2,18)$.

QUADRO 6: Correlação entre a Medicação Efetuada e as Hiperplasias Gengivais Observadas

\begin{tabular}{|c|c|c|c|}
\hline $\begin{array}{c}\text { NÚMERO } \\
\text { DE CRIANÇAS }\end{array}$ & $\begin{array}{l}\text { MEDICAÇÃO } \\
\text { EFETUADA }\end{array}$ & $\begin{array}{l}\text { NÚMERO DE } \\
\text { HIPERPLASIAS }\end{array}$ & $\begin{array}{c}\text { PERCENTAGEM } \\
\%\end{array}$ \\
\hline 8 & $\begin{array}{l}\text { Fenitoína } \\
\text { (Hidantina) }\end{array}$ & 5 & 62,50 \\
\hline 0 & $\begin{array}{l}\text { Valproato de Sódio } \\
\text { (Diplexil) }\end{array}$ & 0 & 0,00 \\
\hline 5 & $\begin{array}{l}\text { Carbamazepina } \\
\text { (Tegretol) }\end{array}$ & 0 & 0,00 \\
\hline 3 & $\begin{array}{l}\text { Clonazepam } \\
\text { (Rivotril) }\end{array}$ & 0 & 0,00 \\
\hline 0 & $\begin{array}{l}\text { Primidona } \\
\text { (Mysoline) }\end{array}$ & 0 & 0,00 \\
\hline 8 & $\begin{array}{l}\text { Fenobarbital } \\
\text { (Luminal) }\end{array}$ & 0 & 0,00 \\
\hline 3 & $\begin{array}{c}\text { Diplexil + } \\
\text { Rivotril }\end{array}$ & 1 & 33,33 \\
\hline 3 & $\begin{array}{c}\text { Tegretol + } \\
\text { Mysoline }\end{array}$ & 2 & 66,66 \\
\hline 2 & $\begin{array}{c}\text { Tegretol + } \\
\text { Hidantina }\end{array}$ & 1 & 50,00 \\
\hline 3 & $\begin{array}{c}\text { Tegretol + } \\
\text { Rivotril }\end{array}$ & 2 & 66,66 \\
\hline 6 & $\begin{array}{c}\text { Diplexil + } \\
\text { Fenobarbital }\end{array}$ & 2 & 33,33 \\
\hline 3 & $\begin{array}{r}\text { Diplexil + } \\
\text { Tegretol }\end{array}$ & 0 & 0,00 \\
\hline
\end{tabular}


Nas crianças epilépticas sem hiperplasia gengival, o valor médio foi de 2,44 ..

Do registro e análise da dieta, constatamos que $60 \%$ das crianças que observamos com hiperplasia gengival, fazem dieta mole e hipercalórica, fundamentalmente com predominância de farinhas lácteas, por se tratar de deficientes profundos com grande diminuição da função mastigatória.

Pudemos observar ainda que enquanto $60 \%$ das crianças com hiperplasia gengival, iniciaram terapêutica medicamentosa antes dos 12 meses de idade, apenas $21,4 \%$ das que não desenvolveram hiperplasia gengival, iniciaram também medicaçăo anti-epiléptica antes de 1 ano de idade.

A orientação terapêutica efetuada a estes pacientes consistiu na instituição de medidas de higiene oral, na correçāo da dieta e na proposta de alteração medicamentosa aos colegas Neurologistas, sempre que a mesma se nos afigurava possível. (Quadro 7).

Com estas três medidas, associadas a destartarizaçōes periódicas e à remoção dos focos sépticos orais, conseguiu-se controlar a grande maioria das situaçōes de hiperplasia gengival. Apenas houve necessidade de recorrer à Cirurgia (gengivectomia) em duas crianças.

\section{CONCLUSÕES}

Há alteraçōes gengivais em crianças a efetuar medicaçăo anti-epiléptica. A Hiperplasia Gengival encontra-se associada principalmente à Fenitoína e à Primidona.

Apresenta íntima relaçäo com a deficiente higiene oral e encontramos como fator adjuvante a dieta mole.

QUADRO 7:

\begin{tabular}{|c|c|c|}
\hline $\begin{array}{c}\text { ORIENTACC̃O } \\
\text { TERAPEUUICA }\end{array}$ & $\begin{array}{c}\text { No: DE } \\
\text { CASOS }\end{array}$ & $\begin{array}{c}\text { PERCENT AGEM } \\
(\%)\end{array}$ \\
\hline Melhoria de Higiene Oral & 50 & 98,03 \\
Correção da Dieta & 10 & 19,60 \\
Alteração da Terapêutica & 2 & 3,92 \\
Cirurgia Gengival & 2 & 3,92 \\
\hline
\end{tabular}

\section{REFERÊNCIAS BIBLIOGRÁFICAS}

1. HARRISON, Medicina interna, v. 2, p. 2243, 10. ed., Guanabara Koogan, Brasil, 1984.

2. MARCUS, A.; KRUPP, MILTON J., CHATTON. Diagnóstico clínico e tratamento. p. 584-589, 21. ed., 1983.

3. GOTH, A., Farmacologia médica, p. 273-278, Editorial Interamericana, S.A., 1979.

4. MALCOM, A. LYNCH, Burket's Oral Medicine, 7. ed., J.B. Lippincort Company. Philadelph, 1977.

5. LINDHE, J., Tratado de periodontologia clínica, p. 223-224, Editora Guanabara S.A., Brasil, 1988.

6. SHAFER, WILLIAM G., Patologia bucal, $p$. 727-728, 4. ed. Interamericana, 1985.

\begin{tabular}{|c|c|c|c|c|c|c|}
\hline R. Fac. Odontol. & Porto Alegre & V.32 & N. 1 & p.31-31 & JULHO & 1991 \\
\hline
\end{tabular}

